\title{
Impact of Subthalamic Deep Brain Stimulation Frequency on Upper Limb Motor Function in Parkinson's Disease
}

\author{
Sheikh Momin ${ }^{\mathrm{a}}$, Philipp Mahlknecht ${ }^{\mathrm{a}, \mathrm{b}}$, Dejan Georgiev ${ }^{\mathrm{a}, \mathrm{c}}$, Thomas Foltynie ${ }^{\mathrm{a}}$, Ludvic Zrinzo ${ }^{\mathrm{a}}$, \\ Marwan Hariz ${ }^{\mathrm{a}}$, Andre Zacharia ${ }^{\mathrm{a}, \mathrm{d}}$ and Patricia Limousin ${ }^{\mathrm{a}, *}$ \\ ${ }^{a}$ Sobell Department of Motor Neuroscience, UCL Institute of Neurology, National Hospital \\ for Neurology and Neurosurgery, London, UK \\ ${ }^{\mathrm{b}}$ Department of Neurology, Innsbruck Medical University, Innsbruck, Austria \\ ${ }^{\mathrm{c}}$ Department of Neurology, University Medical Centre Ljubljana, Slovenia \\ ${ }^{\mathrm{d}}$ Department of Neurology, University Hospitals of Geneva, Switzerland
}

Accepted 24 January 2018

\begin{abstract}
.
Background: Whilst changes in the frequency of subthalamic deep brain stimulation (STN-DBS) have been proposed to improve control of tremor or axial motor features in Parkinson's disease (PD), little is known about the effects of frequency changes on upper limb motor function, particularly bradykinesia.

Objective: To investigate the acute effects of various STN-DBS frequencies $(40-160 \mathrm{~Hz}, 40 \mathrm{~Hz}$ intervals) on upper limb motor function.

Methods: We carried out a randomised, double-blind study on 20 PD patients with chronic STN-DBS using the Simple and Assembly components of the Purdue Pegboard (PP) test and a modified upper limb version of the UPDRS-III (UL-UPDRS-III). Results: There was no significant effect of frequency on bradykinesia on the Simple PP task or the UL-UPDRS-III. There was an effect of frequency on the Assembly PP score when comparing all frequencies $(p=0.019)$ and between $80 \mathrm{~Hz}$ and $130 \mathrm{~Hz}(p=0.007)$, with lower frequencies yielding a better performance. Rigidity and Tremor scores were significantly reduced with higher $(>80 \mathrm{~Hz})$ compared to lower $(40 \mathrm{~Hz})$ frequencies.

Conclusions: Our findings suggest that a wide range of frequencies are efficacious in improving acute upper-limb motor function. Reducing the frequency of stimulation down to $80 \mathrm{~Hz}$ is safe and has a similar clinical effect to higher frequencies. Therefore, a wider range of frequencies are available when it comes adjusting patients' acute settings without the risk of worsening bradykinesia.
\end{abstract}

Keywords: Bradykinesia, deep brain stimulation, Parkinson's disease, rigidity, subthalamic nucleus, tremor

\section{INTRODUCTION}

Deep brain stimulation of the subthalamic nucleus (STN-DBS) has been an established therapy for

\footnotetext{
${ }^{*}$ Correspondence to: Professor Patricia Limousin, $\mathrm{MD}, \mathrm{PhD}$, Unit of Functional Neurosurgery, UCL Institute of Neurology, National Hospital for Neurology and Neurosurgery, Box 146, Queen Square, London WC1N 3BG, UK. Tel.: +44 203448 8723; E-mail: p.limousin@ucl.ac.uk.
}

over 20 years in advanced Parkinson's disease (PD) patients who have developed fluctuations in the motor response to levodopa-based therapy and can produce a dramatic improvement in cardinal PD symptoms $[1,2]$. The electrical parameters for DBS systems have largely been empirically derived [3]. However, one double-blind study in which frequency, voltage and pulse width were systematically manipulated found that voltage had the greatest influence on motor 
symptoms and that voltages $\geq 3 \mathrm{~V}$ and frequencies $\geq 130 \mathrm{~Hz}$ led to the greatest symptomatic improvement in PD patients [4], supporting current clinical practice.

Nevertheless, high frequency (HFS, defined as frequency $>100 \mathrm{~Hz}$ ) DBS can lead to notable side effects largely axial in nature, such as gait freezing, swallowing disturbance and decreased verbal fluency [5]. Low frequency stimulation $(\mathrm{LFS},<100 \mathrm{~Hz})$ can also benefit axial motor symptoms [6], but its effects on limb motor function have not been systematically assessed. Therefore, we sought to investigate the effect of STN-DBS frequency on upper limb motor function in general, and limb bradykinesia in particular. We carried out a randomised, double-blind study to assess how frequencies ranging from $40-160 \mathrm{~Hz}$ in $40 \mathrm{~Hz}$ intervals, affected relief of these PD symptoms in the acute setting.

\section{METHODS}

20 patients with idiopathic PD (as defined per the United Kingdom Brain Bank Criteria) with chronic bilateral STN-DBS stimulation ( $>6$ months) were selected for the study. The study protocol was approved by the local ethics committee and all participants gave informed written consent as per the declaration of Helsinki. Inclusion criteria were applied to PD patients with chronic STN-DBS as follows: 1) Participants were cognitively competent based on Mini-Mental State Examination (MMSE) $>24$ and frontal assessment battery (FAB) $>12 ; 2$ ) Motor symptoms of PD (for instance, tremor) were not severe enough to completely compromise task performance; 3) There was no acute concomitant disease and no other neurological conditions.

Participants were assessed OFF-medication following at least 12 hours of medication withdrawal overnight. After an assessment at the participants' baseline frequency $(130 \mathrm{~Hz})$, frequencies from $40-160 \mathrm{~Hz}$ in $40 \mathrm{~Hz}$ intervals were used, with the order of frequencies randomised using a random number generator. Each participant served as his/her own control. Both the participant and examiner (SM) were blinded to the frequencies. For each trial, a neurologist with expertise in movement disorders (AZ, DG, or PM) programmed the frequency at least 10 minutes before reassessment; we felt this interval to be an appropriate compromise between being able to assess for a meaningful clinical effect in the new frequency whilst minimising the length of any sig- nificantly discomforting or intolerable side-effects. The Total Electrical Energy Delivered (TEED) was kept constant at the baseline level across frequencies by adjusting the voltage using an established formula [7].

\section{Experimental tasks}

In order to minimise the burden of assessment, we decided to focus on assessing upper limb function, given that it is the standard method of assessing the clinical effect of DBS [8]. Two validated tasks were used to assess upper limb motor function, particularly bradykinesia: the Purdue Pegboard task (PP) [9] and a modified Unified PD Rating Scale motor scale including all items concerning upper limb motor function (UL-UPDRS-III). For the PP, both the Simple and Assembly tasks were used; in the Simple task, participants were scored on the number of pegs placed into the pegboard in 30 seconds, with left hand, right hand and then both hands. In the Assembly task, participants assembled a peg sequentially with four components using both hands, and were scored by the number of pegs fully assembled ( 1 point) and the number of partially assembled pegs in the correct sequence ( 0.5 points). Participants were permitted to briefly practise the tasks beforehand for familiarisation. The UL-UPDRS-III comprised measures of upper body motor function: distal limb bradykinesia (items 23-25, 6 measures), rest tremor (item 20, 3 measures) and rigidity (item 22, 3 measures), which were summated into subscales. Each parameter was scored from 0 (normal) to 4 (severe). Examinations were videotaped and UL-UPDRS-III scores were subsequently rated by a separate neurologist blinded to stimulation frequencies (AZ, DG, or PM).

\section{Statistical analysis}

Statistical analysis was carried out using R 3.2.1. [10] Where the data set was normally distributed (assessed by Shapiro-Wilk test), analysis of variance (ANOVA) and paired $t$-tests were used. If data were non-normally distributed, the Kruskal-Wallis test and Wilcoxon Signed Rank Test were used. All data was analysed for significance at the $<0.05$ level. Analyses were made to compare all frequencies $(130 \mathrm{~Hz}$, $40 \mathrm{~Hz}, 80 \mathrm{~Hz}, 120 \mathrm{~Hz}, 160 \mathrm{~Hz}$ ), with post-hoc testing (Bonferroni correction and Tukey test) conducted between all frequencies where a significant difference was found. 


\section{RESULTS}

Characteristics of the 20 participants are provided in Table 1. All 20 participants completed the study, but three participants could not tolerate $40 \mathrm{~Hz}$ due to intolerable side effects, including worsening of parkinsonism (particularly bradykinesia and tremor) and oscillopsia. The results for both experimental tasks along with statistical significance are presented in Table 2.

Regarding the PP, there was a significant difference in the Assembly PP task, but not in the Simple PP task. Post-hoc testing showed that $80 \mathrm{~Hz}$ produced a significantly higher score for the Assembly PP task over the baseline $(130 \mathrm{~Hz})$ frequency $(p=0.007)$ and $40 \mathrm{~Hz}$ $(p=0.04)$, but this was not reproduced in comparisons with $120 \mathrm{~Hz}$ or $160 \mathrm{~Hz}$. In comparison, the Tremor and Rigidity scores for the UL-UPDRS-III reduced significantly with higher experimental frequencies, but there was no significant difference between frequencies for Bradykinesia or Total Score. Post-hoc

Table 1

Characteristics of study participants

\begin{tabular}{lc}
\hline Participant Characteristic $(n=20)$ & Mean $( \pm$ SD $)$ \\
\hline Age (years) & $60.4 \pm 10.0$ \\
Sex & 15 male, 5 female \\
Handedness & 20 right, 0 left \\
PD Duration (years) & $11.8 \pm 4.9$ \\
Duration of DBS therapy (years) & $2.5 \pm 3.0$ \\
Levodopa equivalent dose (mg/day) & $702.4 \pm 304.4$ \\
UPDRS-III (ON-stimulation/ & $15.6 \pm 8.6$ \\
$\quad$ ON-medication) & \\
MMSE & $28.9 \pm 1.5$ \\
FAB & $16.9 \pm 1.4$ \\
\hline
\end{tabular}

UPDRS-III, Unified Parkinson's Disease Rating Scale, Motor Subesection; MMSE, Mini-Mental State Exam; FAB, Frontal Assessment Battery. testing showed that $40 \mathrm{~Hz}$ gave significantly higher Tremor and Rigidity scores, driving the overall significance for these parameters.

\section{DISCUSSION}

In this experimental study, we found no consistent effect of frequency on bradykinesia in the acute setting using two different approaches to measurement. Our modified version of the UPDRS did not reveal a difference between high and low frequencies; this contrasts with two earlier comparable studies, which suggested that high frequencies, particularly those $\geq 130 \mathrm{~Hz}$, are significantly better for reducing bradykinesia [4, 11]. However, another single-blinded study also investigated the effect of frequency on bradykinesia in $6 \mathrm{PD}$ patients and found no significant difference across a range of $0-250 \mathrm{~Hz}$ on bradykinesia [12]. These three studies all used a smaller number of patients than in the present study, and in two of the three studies the TEED was not kept constant, therefore introducing a huge variability in the total current delivered to each participant $[4,11,12]$.

Furthermore, our study found a significant difference in Assembly PP task performance between $80 \mathrm{~Hz}$ and baseline $(130 \mathrm{~Hz})$, whilst $120 \mathrm{~Hz}$ and $160 \mathrm{~Hz}$ produced an improved-though nonsignificant-performance. This suggests a similar benefit at these frequencies on the increased phasic alertness and divided attention required on the Assembly PP task over the Simple PP task [13]. As expected, we found that tremor but also rigidity were significantly better controlled at higher frequencies, which has been demonstrated previously $[3,4]$. Inspecting the post-hoc comparisons, only $40 \mathrm{~Hz}$ was

Table 2

Upper limb motor function according to various frequencies tested

\begin{tabular}{|c|c|c|c|c|c|c|c|}
\hline & & \multicolumn{5}{|c|}{ DBS Frequency $(\mathrm{Hz})$} & \multirow[t]{2}{*}{$p$-value } \\
\hline & & Baseline [130] & 40 & 80 & 120 & 160 & \\
\hline Mean & Left Hand & $8.1 \pm 0.6$ & $7.5 \pm 0.8$ & $7.8 \pm 0.7$ & $8.4 \pm 0.7$ & $8.4 \pm 0.5$ & 0.15 \\
\hline Simple & Right Hand & $9.7 \pm 0.4$ & $9.1 \pm 0.8$ & $10.1 \pm 0.6$ & $9.7 \pm 0.5$ & $10.0 \pm 0.6$ & 0.32 \\
\hline PP Score & Both Hands & $6.1 \pm 0.6$ & $6.1 \pm 0.6$ & $6.7 \pm 0.6$ & $6.1 \pm 0.6$ & $6.1 \pm 0.5$ & 0.22 \\
\hline Assembly PP Score & & $1.9 \pm 0.1$ & $1.9 \pm 0.2$ & $2.3 \pm 0.2$ & $2.2 \pm 0.2$ & $2.1 \pm 0.2$ & $0.019^{1}$ \\
\hline Mean UL-UPDRS & Bradykinesia (0-24) & $7.6 \pm 0.9$ & $9.2 \pm 1.6$ & $8.3 \pm 1.3$ & $9.4 \pm 1.1$ & $8.3 \pm 1.1$ & 0.69 \\
\hline \multirow[t]{3}{*}{-III score } & Tremor $(0-12)$ & $0.8 \pm 0.3$ & $2.0 \pm 0.5$ & $1.0 \pm 0.3$ & $0.4 \pm 0.2$ & $0.4 \pm 0.2$ & $<0.001^{2}$ \\
\hline & Rigidity (0-12) & $3.2 \pm 0.5$ & $4.7 \pm 0.7$ & $3.4 \pm 0.6$ & $2.9 \pm 0.4$ & $3.4 \pm 0.5$ & $0.029^{3}$ \\
\hline & Total $(0-48)$ & $11.60 \pm 1.40$ & $15.8 \pm 2.4$ & $12.7 \pm 1.9$ & $12.7 \pm 1.4$ & $12.0 \pm 1.4$ & 0.37 \\
\hline
\end{tabular}

PP, Purdue Pegboard; UL-UPDRS-III, Upper Limb Unified Parkinson's Disease Rating Scale, Motor Subsection. All values are mean \pm SD. $P$-values are given for comparisons between all experimental frequencies, with details of post-hoc testing given for significant comparisons. ${ }^{1}$ There was a significant difference between $80 \mathrm{~Hz}$ and $40 \mathrm{~Hz}(0.34,95 \% \mathrm{CI}-0.04-0.73 ; p=0.04)$ and between $80 \mathrm{~Hz}$ and $130 \mathrm{~Hz}(0.35$, 95\% CI: $0.13-0.57 ; p=0.007) .{ }^{2}$ There were significant differences between $40 \mathrm{~Hz}$ and all other experimental frequencies. ${ }^{3} \mathrm{There}$ was a significant difference between $120 \mathrm{~Hz}$ and $40 \mathrm{~Hz}(-1.82,95 \% \mathrm{CI}-3.12--0.54)$. 
associated with significantly higher UL-UPDRS-III scores; indeed, three patients could not complete the experimental tasks whilst on $40 \mathrm{~Hz}$. This resulted from a mixture of parkinsonism caused by the low frequency state and (what appears clinically to be) capsular side effects from the higher voltage required to maintain a constant TEED. Hence, $40 \mathrm{~Hz}$ clearly appears to be unsuitable for use in clinical practice. Comparatively, patients on $80 \mathrm{~Hz}$ DBS experienced effects akin to the higher frequency states in the acute setting. Therefore, categorising frequencies as 'LFS' or 'HFS' using a $100 \mathrm{~Hz}$ threshold may be misleading as this does not always correlate to clinical efficacy.

Although the precise mechanism of DBS is debated $[14,15]$, our findings support the theory that HFS DBS overrides pathological low frequency beta oscillatory activity $(11 \mathrm{~Hz}-30 \mathrm{~Hz})$ and establishes a more regular firing pattern at a higher prokinetic gamma frequency $(>60 \mathrm{~Hz})$ in dopaminergic pathways for symptoms such as bradykinesia and rigidity; simultaneously, in the low-frequency oscillations of tremor pathways, HFS may hold neurons in a refractory state and thus help alleviate tremor [16].

It is important to consider several limitations to our study. Firstly, 20 patients were selected for the study, and only 17 could tolerate all experimental frequencies. Although this is the largest study of its kind $[4,11,12]$, it is likely to be still underpowered to detect subtle differences. Secondly, it may be argued that the interval between changing experimental frequencies (10 minutes) is too short. This strategy was applied as such for several reasons: 1) to ensure that residual effects of the preceding frequency do not influence performance at subsequent frequencies, due to cumulative patient fatigue. Temperli and colleagues reported that $75 \%$ of the worsening of the UPDRS-III scores occurred from 15 minutes onwards after STN-DBS is 'switched off' [17], whilst Krack and colleagues suggested that when STN-DBS is switched on, the full effect on motor function usually occurs within a few seconds, though this can be variable [8]. This suggests that the majority of our findings would have remained consistent with a longer time interval. 2) It is challenging to expose patients to longer between-trial intervals as they attended the study in an off medication state. 3) Our randomisation strategy was designed in order to mitigate any repetition effect and carry over effect. Thirdly, to assess the clinical applicability of our findings, a study assessing whether these experimental frequencies can have a comparable chronic sustainable effect as compared to baseline over a longer time period should be conducted, perhaps also assessing the effect of frequency (especially LFS) on other PD symptoms. These assessments were beyond the scope of this acute study.

Moreover, though there has been debate about the usefulness of the TEED [18], we decided to keep the TEED constant in our study. In our experience, patients who benefited from a lower frequency also had a higher voltage programmed according to TEED. This experience was also shared by others, as described by Zibetti and colleagues in a retrospective study on LFS [19]. However, this phenomenon is not universal, and the reasons why some patients do not benefit are not well understood and require further investigation.

A clear strength of this study was its randomized double-blind design, which minimised several possible confounding factors. Each participant served as their own control, with their performance in the experimental frequencies compared to their baseline score, thus age and sex differences, practice effect and fatigue between participants on task performance was minimised.

In conclusion, there was no significant difference between different frequencies of STN-DBS on upper-limb bradykinesia in PD when tested acutely, suggesting that a wider range of frequencies could be used to tackle bradykinesia providing total electrical energy delivered is maintained. Although HFS appears better for tremor control, $80 \mathrm{~Hz}$ may be an option in well-selected patients without the risk of worsening bradykinesia, particularly if gait, swallow or speech problems coexist. Further studies focusing on longer-term follow-up assessing an expanded range of $\mathrm{PD}$ symptoms at these frequencies are warranted in order to demonstrate the clinical applicability of these findings.

\section{ACKNOWLEDGMENTS}

This study was funded by a grant from the Brain Research Trust (BRT). The Unit of Functional Neurosurgery, UCL Institute of Neurology, Queen Square, London is also supported by the Parkinson's Appeal and the Sainsbury Monument Trust. SM carried out this project as part of an intercalated degree in Neuroscience, which was supported by the Clothworkers' Foundation, UK. PM was supported by a research grant from the Austrian Society of Neurology. AZ was supported by the Fond de Perfectionnement des Hôpitaux Universitaires de 
Genève and by the Swiss National Science Foundation project: P2GEP3_151597.

\section{CONFLICT OF INTEREST}

The authors report no conflict of interest related to the manuscript.

\section{FINANCIAL DISCLOSURES}

MH has received travel expenses and honoraria for speaking at meetings from Medtronic and Boston Scientific.

\section{REFERENCES}

[1] Hamani C, Richter E, Schwalb JM, Lozano AM (2005) Bilateral subthalamic nucleus stimulation for Parkinson's disease: A systematic review of the clinical literature. Neurosurgery 56, 1313-1321; discussion 1321-1314.

[2] Aviles-Olmos I, Kefalopoulou Z, Tripoliti E, Candelario J, Akram H, Martinez-Torres I, Jahanshahi M, Foltynie T, Hariz M, Zrinzo L, Limousin P (2014) Long-term outcome of subthalamic nucleus deep brain stimulation for Parkinson's disease using an MRI-guided and MRI-verified approach. J Neurol Neurosurg Psychiatry 85, 1419-1425.

[3] Limousin-Dowsey P, Tisch S (2009) DBS stimulator programming. In Deep brain stimulation, Bain PG, ed. Oxford University Press, Oxford.

[4] Moro E, Esselink RJ, Xie J, Hommel M, Benabid AL, Pollak P (2002) The impact on Parkinson's disease of electrical parameter settings in STN stimulation. Neurology 59, 706-713.

[5] Wojtecki L, Timmermann L, Jorgens S, Sudmeyer M, Maarouf M, Treuer H, Gross J, Lehrke R, Koulousakis A, Voges J, Sturm V, Schnitzler A (2006) Frequency-dependent reciprocal modulation of verbal fluency and motor functions in subthalamic deep brain stimulation. Arch Neurol 63, 1273-1276.

[6] Xie T, Vigil J, MacCracken E, Gasparaitis A, Young J, Kang W, Bernard J, Warnke P, Kang UJ (2015) Low-frequency stimulation of STN-DBS reduces aspiration and freezing of gait in patients with PD. Neurology 84, 415-420.
[7] Koss AM, Alterman RL, Tagliati M, Shils JL (2005) Calculating total electrical energy delivered by deep brain stimulation systems. Ann Neurol 58, 168; author reply 168169.

[8] Krack P, Fraix V, Mendes A, Benabid AL, Pollak P (2002) Postoperative management of subthalamic nucleus stimulation for Parkinson's disease. Mov Disord 17(Suppl 3), S188-S197.

[9] Vingerhoets FJ, Schulzer M, Calne DB, Snow BJ (1997) Which clinical sign of Parkinson's disease best reflects the nigrostriatal lesion? Ann Neurol 41, 58-64.

[10] Team RDC (2008) R Foundation for Statistical Computing, Vienna, Austria.

[11] Limousin P, Pollak P, Benazzouz A, Hoffmann D, Broussolle E, Perret JE, Benabid AL (1995) Bilateral subthalamic nucleus stimulation for severe Parkinson's disease. Mov Disord 10, 672-674.

[12] Huang H, Watts RL, Montgomery EB Jr (2014) Effects of deep brain stimulation frequency on bradykinesia of Parkinson's disease. Mov Disord 29, 203-206.

[13] Strenge H, Niederberger U, Seelhorst U (2002) Correlation between tests of attention and performance on grooved and Purdue pegboards in normal subjects. Percept Mot Skills 95, 507-514.

[14] McIntyre CC, Savasta M, Walter BL, Vitek JL (2004) How does deep brain stimulation work? Present understanding and future questions. J Clin Neurophysiol 21, 40-50.

[15] Wichmann T, DeLong MR (2016) Deep brain stimulation for movement disorders of basal ganglia origin: Restoring function or functionality? Neurotherapeutics 13, 264-283.

[16] Birdno MJ, Grill WM (2008) Mechanisms of deep brain stimulation in movement disorders as revealed by changes in stimulus frequency. Neurotherapeutics 5, 14-25.

[17] Temperli P, Ghika J, Villemure JG, Burkhard PR, Bogousslavsky J, Vingerhoets FJ (2003) How do parkinsonian signs return after discontinuation of subthalamic DBS? Neurology 60, 78-81.

[18] Fasano A, Lozano AM (2014) The FM/AM world is shaping the future of deep brain stimulation. Mov Disord 29, 161163.

[19] Zibetti M, Moro E, Krishna V, Sammartino F, Picillo M, Munhoz RP, Lozano AM, Fasano A (2016) Low-frequency subthalamic stimulation in Parkinson's disease: Long-term outcome and predictors. Brain Stimul 9, 774-779. 